\title{
Transient Storage of a Tactile Memory Trace in Primary Somatosensory Cortex
}

\author{
Justin A. Harris, ${ }^{1}$ Carlo Miniussi, ${ }^{2}$ Irina M. Harris, ${ }^{1}$ and Mathew E. Diamond ${ }^{1}$ \\ ${ }^{1}$ Cognitive Neuroscience Sector, International School for Advanced Studies (SISSA), Trieste 34014, Italy, and ${ }^{2}$ Clinical \\ Neurophysiology Laboratory, Istituto di Ricovero e Cura a Carattere Scientifico San Giovanni di Dio, 25125 Brescia, Italy
}

\begin{abstract}
Working memory is known to involve prefrontal cortex and posterior regions of association cortex (e.g., the inferior temporal lobes). Here, we investigate the potential role of primary somatosensory cortex (SI) in a working memory task with tactile stimuli. Subjects were required to compare the frequencies of two vibrations separated by a retention interval of $1500 \mathrm{msec}$. Their performance was significantly disrupted when we delivered a pulse of transcranial magnetic stimulation (TMS) to the contralateral SI early (300 or $600 \mathrm{msec}$ ) in the retention interval. TMS did not affect tactile working memory if delivered to con-
\end{abstract}

tralateral SI late in the retention interval (at 900 or $1200 \mathrm{msec}$ ), nor did TMS affect performance if delivered to the ipsilateral SI at any time point. Primary sensory cortex thus seems to act not only as a center for on-line sensory processing but also as a transient storage site for information that contributes to working memory.

Key words: somatosensory cortex; working memory; flutter vibration; transcranial magnetic stimulation; immediate memory; somatotopic
Working memory refers to the ability to hold and manipulate information for short periods (on the order of seconds) and to update the information as required by moment-to-moment demands. As such, it plays an important role in many cognitive processes, functioning as an interface between perception, attention, memory, and action (Baddeley, 1996). Studies investigating the neural basis of working memory have uncovered an important role for prefrontal cortex in the executive monitoring of mnemonic information. For example, brain imaging studies in humans and electrophysiological recordings in monkeys have shown that populations of neurons in the prefrontal cortex become active while subjects perform working memory tasks (for review, see Goldman-Rakic, 1996; Ungerleider et al., 1998; Fuster, 2001), and lesions to areas of prefrontal cortex disrupt working memory function (for review, see Petrides, 2000b). Similar experimental approaches have provided evidence that posterior regions of cortex, such as the inferior temporal lobe, also contribute to working memory, possibly as one location where information is maintained during the retention period (Fuster and Jervey, 1981; Miyashita and Chang, 1988; Postle et al., 1999; Petrides, 2000a). Working memory for perceptual information has thus come to be viewed as a process in which multiple, widely distributed cortical regions interact to hold a memory trace across short delays (Fuster, 2001). Recent findings suggest that primary sensory cortex may also be part of this network when the task requires retention of information of a sensory rather than semantic or categorical quality. Specifically, several studies with monkeys have observed neuronal activity in primary somatosensory (SI) or

\footnotetext{
Received July 8, 2002; revised July 2, 2002; accepted July 11, 2002.

This study was supported by a fellowship from the Italian Ministry of Universities and Scientific and Technological Research (J.A.H.) and grants from the Telethon Foundation, Consiglio Nazionale delle Ricerche, Ministero della Sanità, Ministero dell'Istruzione, Università e Ricerca, and the James S. McDonell Foundation.

Correspondence should be addressed to Dr. Justin Harris, School of Psychology, University of Sydney, New South Wales 2006, Australia. E-mail: justinh@psych.usyd.edu.au.

Copyright (C) 2002 Society for Neuroscience $0270-6474 / 02 / 228720-06 \$ 15.00 / 0$
}

visual (VI) cortex that is correlated with working memory for tactile or visual information (Zhou and Fuster, 1996, 2000; Super et al., 2001). We have obtained psychophysical evidence consistent with a role for SI in tactile working memory in humans (Harris et al., 2001b). Here, we describe two experiments that test the proposal that neuronal activity in primary sensory cortex can constitute an essential part of the short-term memory trace.

In both experiments, human subjects were required to perform a working memory task in which they compared two vibrotactile stimuli. Experiment 1 examined whether subjects were more accurate when the two stimuli were presented to the same finger versus when they were presented to fingers on different hands. This would imply a role for SI, because neurons in SI have receptive fields confined to the contralateral side of the body (Penfield and Rasmussen, 1950; Maldjian et al., 1999; Francis et al., 2000; Shoham and Grinvald, 2001), and the hand representation in area $3 \mathrm{~b}$ of SI is acallosal (Jones and Powell, 1969; Killackey et al., 1983) and therefore could not support comparisons across the body midline. To test directly whether SI contributes to the vibrotactile working memory task, experiment 2 examined the effect of applying transcranial magnetic stimulation (TMS) to SI. TMS disrupts ongoing neuronal activity in a localized area of cortex by briefly inducing an electrical field in the tissue below the magnetic coil. The timing of stimulation can be varied during the execution of a task to demonstrate the time course of the involvement of a specific cortical area in that cognitive process (Hallett, 2000; Pascual-Leone et al., 2000). Because we were interested to know when SI contributes to tactile working memory, we applied TMS at different times across the retention interval (Fig. 1).

\section{MATERIALS AND METHODS}

Experiment 1. We asked eight right-handed subjects (five men and three women) between 23 and 34 years of age (mean, 27 years) to compare the frequency of two vibrations delivered to a fingertip. The vibrations were produced using piezoelectric wafers (Morgan Matroc, Bedford, $\mathrm{OH}$ ) individually driven by $80 \mathrm{~V}$ pulses from custom-built amplifiers controlled by a computer running LabVIEW (National Instruments, Austin, 


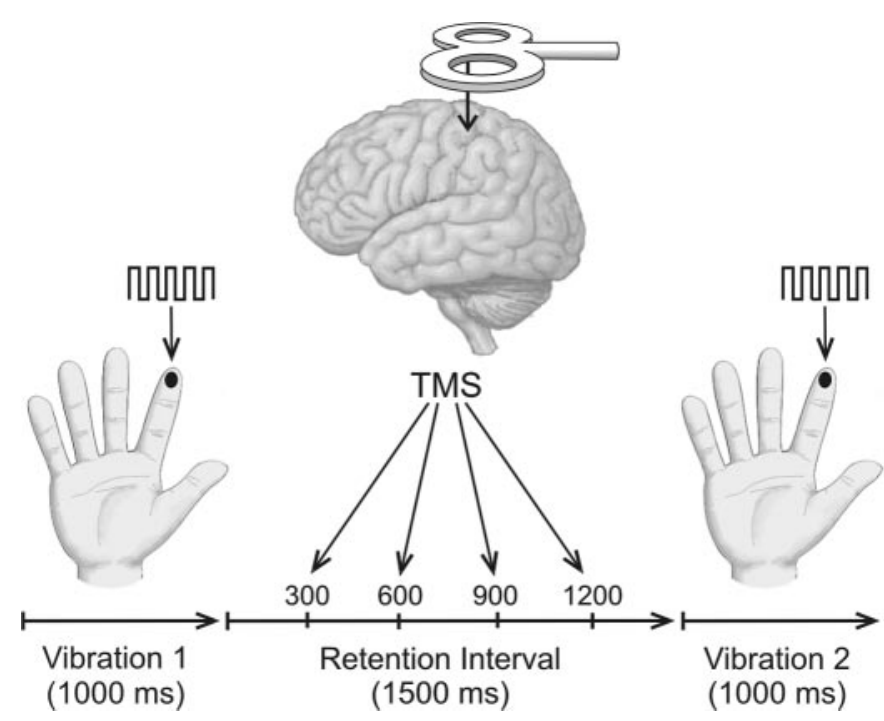

Figure 1. Summary of the procedure for experiments using TMS. Subjects felt two 1000 -msec-long vibrations, separated by a 1500 msec retention interval during which they received a single pulse of TMS. TMS was delivered either $300,600,900$, or $1200 \mathrm{msec}$ after the end of the first vibration $(1200,900,600$, or $300 \mathrm{msec}$ before the start of the second vibration). TMS was applied to the left or right SI, and the vibrations were presented to the left or right index finger. Thus, on half the trials TMS was applied to the SI contralateral to the vibrations, and on the remaining trials, TMS was applied ipsilateral to the vibrations.

TX). For a detailed description of the apparatus, see Harris et al. (2001b). Each vibration was $1000 \mathrm{msec}$ long and consisted of a square wave of fixed amplitude $(250 \mu \mathrm{m})$ and rise time $(5 \mathrm{msec})$. The frequency, measured as the number of deflections per second, was always an even number in the range of $16-24 \mathrm{~Hz}$. The frequency of the two vibrations to be evaluated always differed by $2 \mathrm{~Hz}$, but the specific frequencies varied across trials, forcing subjects to compare the two vibrations rather than make a categorical judgment about one of them (Hernández et al., 1997).

The two vibrations were separated by an interval of $300,600,900$, or $1200 \mathrm{msec}$. There were 80 trials for each retention interval (a total of 320 trials in the experiment). On half of these trials ( 40 per interval), the two vibrations were presented to the same index finger (both on the left or both on the right, counterbalanced), and on the remaining trials they were presented to opposite index fingers (left followed by right or right followed by left, counterbalanced). In each trial, the subjects had to decide whether the second vibration was of higher or lower frequency than the first. Trials from each of the eight conditions (four intervals times two locations) were randomly intermixed, and subjects did not know in advance what the next trial would be. Because neurons in SI have unilateral receptive fields (Penfield and Rasmussen, 1950; Maldjian et al., 1999; Francis et al., 2000; Shoham and Grinvald, 2001), a role for SI can be inferred if subjects are more accurate with same-finger comparisons than opposite-finger comparisons. In contrast, equivalent performance would imply an exclusive role for cortical areas, such as the secondary somatosensory cortex (SII), that possess bilateral receptive fields and strong callosal connections and thus can support comparisons across the body midline (Whitsel et al., 1969; Robinson and Burton, 1980; Killackey et al., 1983; Francis et al., 2000; Disbrow et al., 2001; Ruben et al., 2001).

Experiment 2. We tested 14 right-handed subjects (11 men and 3 women) between 23 and 38 years of age (mean, 31 years). They compared the frequency of two vibrations presented to the same finger, either both on the left index finger or both on the right index finger (for summary of the experimental design, see Fig. 1). The vibrations were separated by $1500 \mathrm{msec}$. During this retention interval, a single $0.25 \mathrm{msec}$ pulse of TMS was delivered to the hand area of SI. Because we were interested to know when SI contributes to holding the tactile working memory trace, we used TMS to track its involvement at different times (for a summary of the experiment, see Fig. 1). To this end, we delivered single TMS pulses at discrete time points $(300,600,900$, and $1200 \mathrm{msec})$ after the end of the first vibration. We chose not to use repetitive TMS (which consists of a train of magnetic pulses) because a single pulse affords much more precise temporal resolution. TMS was delivered using a Magstim (Whitland, UK) rapid magnetic stimulator with a figure-eight (double $70 \mathrm{~mm}$ ) coil, which can induce a maximum magnetic field of 2.2 tesla at the scalp site. Individual resting excitability thresholds of stimulation were previously determined by stimulating the left motor cortex and measuring the amplitude of contractions evoked in the contralateral first interosseus dorsalis muscle by a single TMS pulse. During all subsequent experimental trials, the stimulation intensity was set at $110 \%$ of this threshold for each subject. The mean intensity used during the experiments was $69 \%$ of maximal output, well within safety guidelines issued by the National Institute of Neurological Disorders and Stroke (Wassermann, 1998).

The appropriate location for stimulating the hand area of SI was identified for each subject as the site at which tactile extinction could be most readily obtained. Thus, before beginning the experimental trials, the subject performed a tactile detection task while single TMS pulses were delivered at different positions $\sim 5 \mathrm{~mm}$ posterior to the position at which the motor excitability threshold was obtained. The subject sat with each index finger resting on a piezoelectric wafer, and in each trial, a single 4 msec deflection was presented at one or both wafers (the amplitude of the deflection was set just above the subject's detection threshold, determined earlier). The subject stated whether he or she felt a deflection on the left finger, right finger, or both fingers. On each trial, a single TMS pulse was presented exactly $20 \mathrm{msec}$ after the deflection, at which time it should disrupt tactile detection (Cohen et al., 1991). By moving the coil between trials, we were able to determine the position and orientation of the coil at which the TMS most reliably interfered with the detection task. The typical position of the virtual cathode of the coil was approximately over C3/C4 in the International 10/20 EEG system, with the handle pointing toward the posterior midline. Once SI was located, the coil was held fixed in this position by an articulated mechanical arm for the 80 trials of the experimental block.

To reduce the number of TMS pulses delivered to each individual, the experiment was split across two equal groups of subjects: for seven subjects, TMS was delivered either 300 or $1200 \mathrm{msec}$ after the end of the first vibration; for the other seven subjects, TMS was delivered 600 or 900 msec after the first vibration. In one block of 80 trials, the TMS coil was positioned over the left SI, and the vibrations were presented to the contralateral or ipsilateral index finger ( 40 trials of each, randomly intermixed within each block). In a separate block of 80 trials, the coil was positioned over the right SI, and again vibrations were presented to the contralateral or ipsilateral finger. The order of the two blocks was counterbalanced between subjects. Thus, each subject was tested with 80 trials in which TMS was delivered contralateral to the vibration and 80 trials in which TMS was ipsilateral to the vibration. In half of these trials, TMS was delivered early in the retention interval (either 300 or 600 $\mathrm{msec}$ ), and in the other half of the trials, TMS was delivered late in the interval (900 or $1200 \mathrm{msec}$ ); again, trials of each type were randomly intermixed. Therefore, each subject received 40 trials in each of the four experimental conditions (early versus late times contralateral versus ipsilateral).

Those subjects tested with TMS at 300 versus $1200 \mathrm{msec}$ were given an additional block of 80 trials with "sham" stimulation in which the coil was positioned over the posterior midline, $10 \mathrm{~cm}$ from the stimulation sites over SI, corresponding to $\mathrm{Pz}$ in the International 10/20 EEG system. The coil was held at a $45^{\circ}$ angle to the skull so that most of the magnetic field would miss the brain but still produce a scalp sensation, and TMS was delivered at 300 or $1200 \mathrm{msec}$ into the retention interval. The order of this block relative to the other two was counterbalanced across subjects.

In both experiments, the recruitment of subjects and all experimental protocols were conducted in accordance with the Declaration of Helsinki and were approved by the institutional Bioethics Committee.

\section{RESULTS}

\section{Experiment 1}

As shown in Figure 2, the subjects' accuracy was above chance for all conditions ( $p<0.0001$, two-tailed $Z$ test). However, for the shorter retention intervals (300 and $600 \mathrm{msec}$ ), their accuracy was significantly higher when the two vibrations were presented to the same finger than when they were presented to opposite fingers ( $p=0.014$ and 0.002 for 300 and $600 \mathrm{msec}$ intervals, respectively, by two-tailed paired Student's $t$ test). Indeed, whereas accuracy 


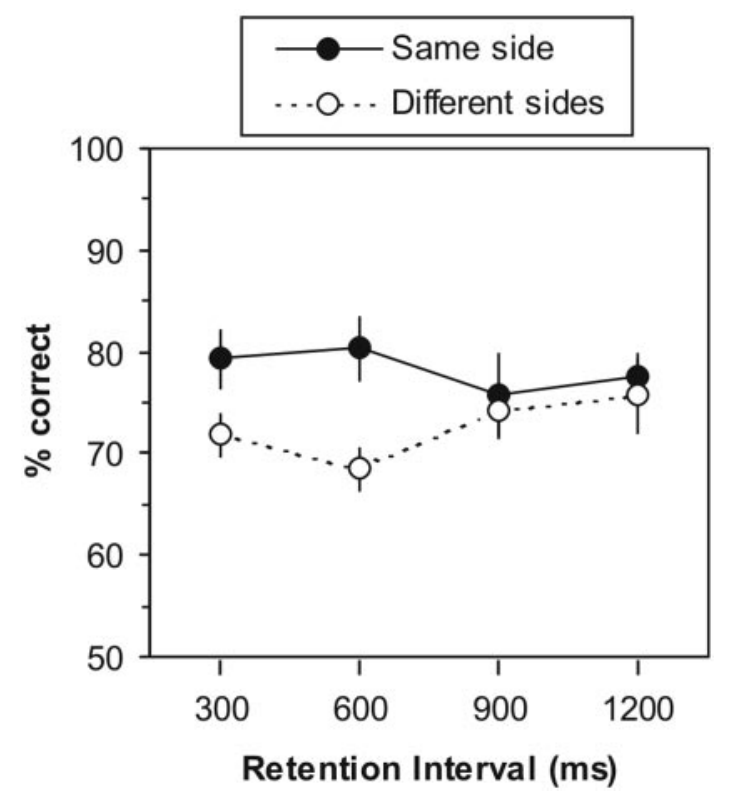

Figure 2. Results of experiment 1, in which subjects compared two vibrations separated by a retention interval of $300,600,900$, or $1200 \mathrm{msec}$. At all intervals, performance was above chance for vibrations presented both on the same side and on opposite sides. However, at the shorter intervals, the subjects were significantly more accurate when the two vibrations were presented on the same side than on different sides $(p<$ $0.05)$. There was no such laterality effect at the longer intervals. Error bars indicate SEM.

with same-finger comparisons was $\sim 30 \%$ above the level expected by chance ( $50 \%$ correct), accuracy for opposite-finger comparisons was only $\sim 20 \%$ above chance. This $10 \%$ difference in accuracy represents a $33 \%$ drop in performance (as a proportion of the accuracy level for single-finger comparisons). We interpret this to mean that at the 300 and $600 \mathrm{msec}$ time points, the memory trace was distributed across both SII (which would allow opposite-finger comparisons) and SI (which would favor same-finger comparisons). In contrast, when the retention interval was 900 or $1200 \mathrm{msec}$, the subjects were equally accurate when comparing vibrations delivered to opposite fingers and when comparing vibrations delivered to the same finger $(p=0.73$ and 0.69 for 900 and $1200 \mathrm{msec}$ intervals, respectively, by two-tailed paired Student's $t$ test). The fact that the initial advantage in making same-finger comparisons disappeared suggests that by $900 \mathrm{msec}$ into the retention interval, no part of the memory trace remained in SI but rather was still held in a cortical area (e.g., SII), where neurons represent tactile stimuli bilaterally. To directly test the hypothesis that tactile information is transiently stored in SI, experiment 2 examined the effect of applying TMS to SI while subjects performed the vibrotactile working memory task.

\section{Experiment 2}

Because tactile stimuli are processed by the contralateral, but not ipsilateral, SI (Penfield and Rasmussen, 1950; Cohen et al., 1991; Maldjian et al., 1999; Francis et al., 2000; Shoham and Grinvald, 2001), we hypothesized that the working memory trace would likewise be held in contralateral SI only. TMS therefore would be expected to affect tactile working memory only when applied contralateral to the stimulus site. Consistent with this model, we found that subjects' accuracy was high during ipsilateral SI stimulation at all time points (between 77 and $81 \%$ correct). Indeed,

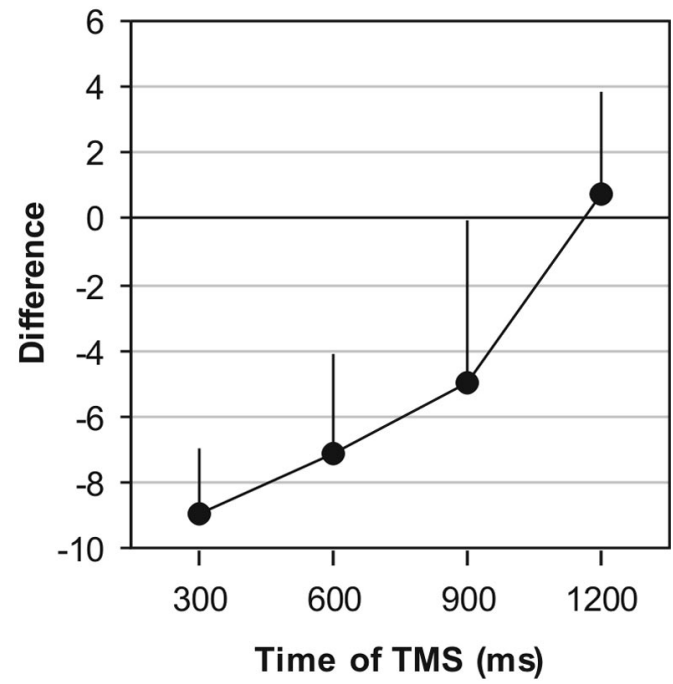

Figure 3. Effects of TMS on vibration discrimination in experiment 2. The plot shows the mean difference in accuracy between trials in which TMS was applied to the contralateral SI and trials in which TMS was applied to the ipsilateral SI. This difference score is significantly below zero when TMS was delivered 300 or $600 \mathrm{msec}$ into the $1500 \mathrm{msec}$ retention interval, but not when TMS was delivered 900 or 1200 msec into the interval. Therefore, TMS disrupted performance when applied to the contralateral SI in the first half of the retention interval. Error bars indicate SEM.

performance with ipsilateral TMS was no different $(p>0.75)$ from performance with a sham TMS procedure in which the pulse was delivered, at either 300 or $1200 \mathrm{msec}$ into the retention interval, to a site on the midline posterior to SI (here, performance ranged from 76 to $80 \%$ correct). Thus, as predicted, disrupting ongoing neuronal activity in the ipsilateral SI did not affect the working memory trace of a tactile stimulus.

In contrast, TMS applied to the contralateral SI did disrupt working memory (accuracy ranged from 70 to $78 \%$ correct). To illustrate the time course of this effect, we calculated for each subject the difference in accuracy between trials on which TMS was applied to the contralateral versus the ipsilateral SI. As shown in Figure 3, these difference scores make it clear that the subjects' accuracy was significantly reduced when TMS was applied to the contralateral SI early in the retention interval $(z=4.56, p<$ 0.00001 , for TMS at $300 \mathrm{msec} ; z=2.34, p<0.01$, for TMS at 600 $\mathrm{msec}$ ). The effect was primarily confined to this time window, because it was no longer statistically reliable when TMS was delivered $900 \mathrm{msec}$ into the retention interval $(z=1.01 ; p=0.16)$, and there was no effect at all when TMS was applied late in the interval (at $1200 \mathrm{msec}, z=-0.22, p=0.59$ ). Thus, tactile working memory was susceptible to disturbance of the contralateral SI across the same interval during which an SI contribution to the task had been inferred from the preceding psychophysical experiment.

The size of effect of TMS on performance is best illustrated by considering accuracy relative to the chance level (50\% correct). When TMS was delivered at $300 \mathrm{msec}$, the subjects' accuracy was $29 \%$ above chance with ipsilateral TMS ("baseline" performance), but it was only $20 \%$ above chance with contralateral TMS. This difference in accuracy represents an effective drop in performance of $30 \%$ (a $9 \%$ decrease relative to the baseline score of $29 \%$ above chance). Similarly, when TMS was presented at 600 msec, accuracy was $31 \%$ above chance with ipsilateral stimulation 

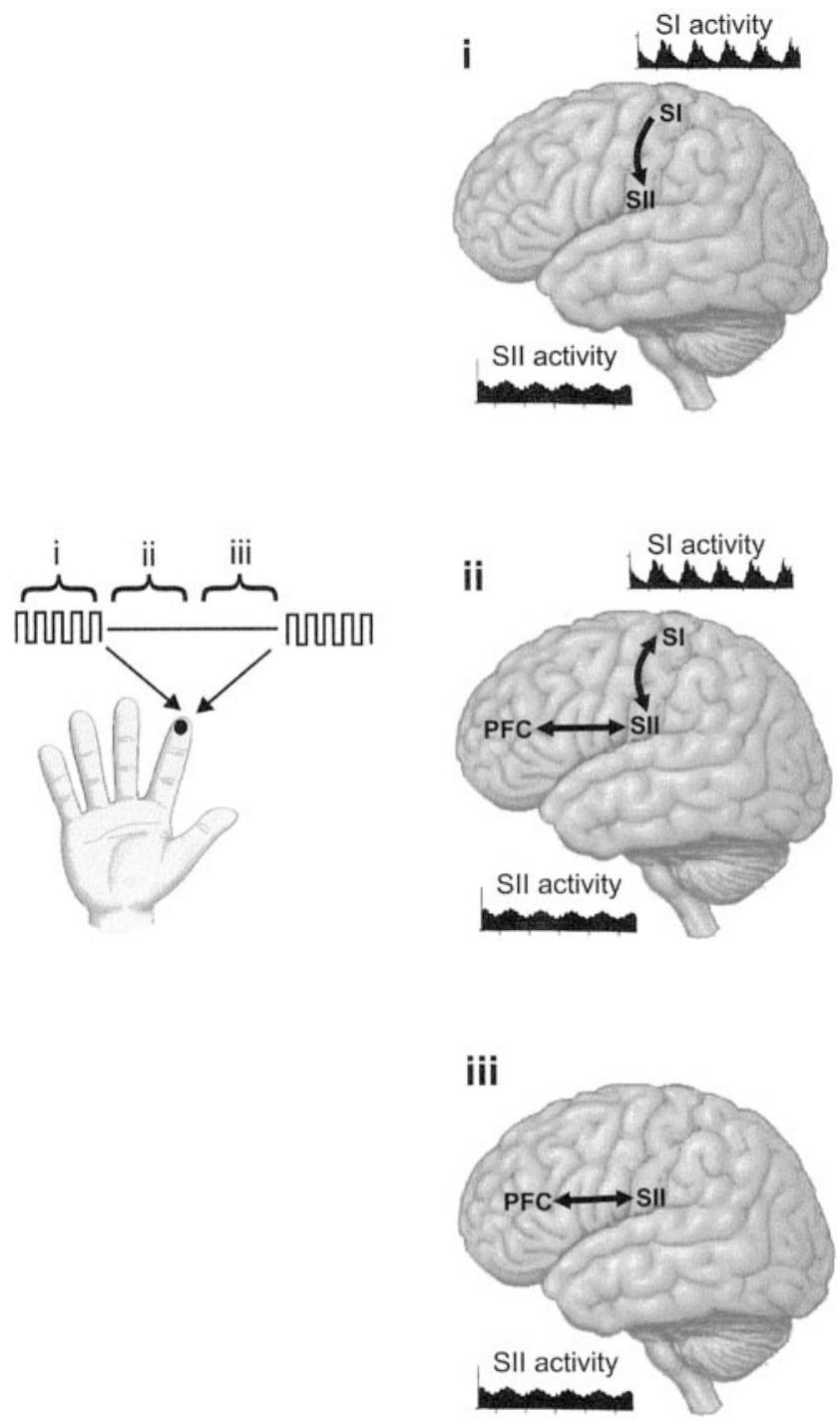

Figure 4. Diagrams showing possible neuronal mechanisms involved in working memory for vibrotactile stimuli. During delivery of the first vibration to a fingertip ( phase $i$ ), the frequency of the vibration is encoded by the firing rate or firing pattern of populations of neurons in primary and secondary somatosensory cortex (SI and $S I I)$. SI neurons fire in phase with the indentation cycle of the vibration, whereas the firing rate of neurons in SII is a monotonic (increasing or decreasing) function of the vibration frequency. Both patterns are depicted here by peristimulus time histograms of neuronal activity, as reported by Salinas et al. (2000). Across the retention interval, subjects must remember the frequency of the first vibration to compare it with the second vibration. We propose that this memory trace is supported initially by ongoing neuronal activity in both SI and SII ( phase ii), but by $900 \mathrm{msec}$ into the interval (phase iii), the memory is no longer held in SI. Populations of neurons in distinct areas of the premotor and prefrontal cortex $(P F C)$ also contribute to sustaining the memory trace, especially toward the latter part of the retention interval (Romo et al., 1999; Hernández et al., 2002).

but $24 \%$ above chance with contralateral stimulation (a drop of $23 \%$ ).

To what extent are the results from the two experiments comparable? If we speculate that the TMS pulse used in experiment 2 eliminated the contribution of SI to working memory, then the resulting performance level should be similar to that in experiment 1 for comparison of opposite-finger stimuli, because SI neurons would be unable to contribute to comparisons across the body midline. In agreement with this reasoning, we note that in experiment 1 at delay intervals of 300 and $600 \mathrm{msec}$, oppositefinger comparisons were $\sim 33 \%$ less accurate than same-finger comparisons, a drop in performance equivalent to that produced by TMS to the contralateral SI in experiment 2 .

\section{DISCUSSION}

We performed two experiments to explore which cortical regions might participate in tactile working memory. Experiment 1 showed that, for short delay intervals (300 or $600 \mathrm{msec}$ ), the subjects' performance in comparing two vibrations was $\sim 33 \%$ better when both stimuli were delivered to the same fingertip than to opposite fingers, indicating that some component of the comparison depended on an area with strictly unilateral receptive fields, such as SI. Experiment 2 showed that disruption of SI functioning early in the delay period (at 300 or $600 \mathrm{msec}$ ) interfered with subsequent working memory performance by $\sim 30 \%$. Therefore, experiments using topography and direct interference by TMS independently lead to the same estimate of when, and by how much, SI contributes to the working memory task.

Neurons in SI respond to low-frequency vibrations by firing in phase with each cycle of the stimulus (Mountcastle et al., 1969, 1990; Hernández et al., 2000; Salinas et al., 2000). Electrical stimulation of SI neurons at a particular frequency produces sensations that monkeys treat as identical to a mechanical vibration of that frequency (Romo et al., 1998, 2000), indicating that SI activity composes an important part of the explicit representation of the vibration. Furthermore, some recent studies have reported that in working memory tasks, neuronal activity in primary sensory cortex can be maintained during the retention interval between two stimuli (Zhou and Fuster, 1996, 2000; Super et al., 2001). The observed correlations between neuronal activity and working memory have led to the inference that maintained neuronal responses may constitute the neural substrate of the working memory trace itself. Here, we have strengthened this proposal by showing that neuronal activity in sensory cortex not only accompanies working memory but also is essential to optimal tactile working memory performance.

Although the results of experiment 1 are consistent with the argument that SI initially contributes to the tactile memory, they could also be interpreted as reflecting a time lag in the shift of attention between hands. Specifically, difficulty in disengaging attention from the finger at which the first vibration was applied could be put forward to explain the subjects' poorer performance on short-interval trials when the two vibrations were presented to opposite fingers. However, recent research indicates that this is not a satisfactory explanation. In light of the effect known as "inhibition of return," whereby people are faster in detecting a tactile cue if it has been preceded by a contralateral cue than if preceded by an ipsilateral one (Röder et al., 2002), the results obtained in experiment 1 would appear to be in spite of attentional effects, rather than because of them.

The effects of TMS reported here cannot be attributed to a direct disruption of SI sensory processing, because TMS applied to the contralateral SI is known to affect processing of tactile stimuli only if delivered within $200 \mathrm{msec}$ of the onset or offset of the stimulus (Cohen et al., 1991). We found diminished working memory performance when TMS was delivered 300 or $600 \mathrm{msec}$ after the end of the first vibration, well outside the time window in which TMS can affect ongoing sensory processing by SI. Indeed, to argue that sensory processing of the vibration in SI was still taking place $>300 \mathrm{msec}$ after the first vibration would imply 
difficulty in comparing vibrations separated by such short retention intervals. The results of experiment 1 show that this is not the case, meaning that the representation of a vibration is fully established within $300 \mathrm{msec}$.

There are numerous reports suggesting that TMS can have long-range actions, affecting neuronal activity in areas that receive projections from the stimulated zone (Paus et al., 1997; Civardi et al., 2001; Strafella et al., 2001; Münchau et al., 2002). In our experiment, was the effect of TMS on tactile working memory the result of a disruption of neuronal activity in SII, the principal downstream target of SI? Although we cannot completely rule out this possibility, there are several arguments against it. First, if SI TMS affected the SII targets of the stimulated site, we would expect to find diminished performance for stimulation of SI ipsilateral to the tactile stimulus site, given that SI projects to both ipsilateral and contralateral SII (Manzoni, 1984). Instead, our experiment showed that the effect of TMS was unilateral, confined to SI contralateral to the tactile stimulus. Second, the effect of TMS was limited to the first $600 \mathrm{msec}$ of the retention interval, corresponding to the time at which subjects were more accurate at comparing vibrations delivered to the same hand than different hands. Indeed, the degree to which TMS affected accuracy matched the difference in accuracy observed for same hand versus different hand comparisons. Thus, the tactile working memory was susceptible to SI TMS during the same interval, and to the same extent, that a separate measure, the topographic distribution, points toward an SI role. Thus, the most parsimonious interpretation of both sets of data is in terms of a role for SI in the task.

We believe that the results are best explained as a disruption of working memory: TMS interfered with the neural mechanisms that support the memory trace of the first vibration. The results thus provide direct support for the claim that a component of the working memory trace for tactile events resides in contralateral SI. Moreover, they show that SI contributed to maintenance of the memory trace for only a limited time $(<1 \mathrm{sec})$, after which the perceptual record appears to have been held beyond this area. Although the present data cannot specify the storage sites outside SI, we emphasize that even across longer delays (1-2 sec), tactile working memory was topographically organized: performance in comparing two vibrations was better when the stimuli were delivered to the same finger, opposite fingers, or neighboring fingers than to fingers separated by greater distances (Harris et al., 2001b). For this reason, we propose that under our experimental conditions the tactile memory trace is initially distributed across both SI and SII but is subsequently limited to SII. This model is illustrated in Figure 4.

What is the nature of the transient memory in SI? One potential account holds that SI maintains an immediate sensory memory, such as the "iconic" and "echoic" memory traces described in visual and auditory modalities (Sperling, 1960; Darwin et al., 1972; Lu et al., 1992). This "echo," however, should not be viewed as a mere sensory aftereffect divorced from the subsequent memory trace. First, in experiment 1, the elevated performance of the task for same-finger comparisons suggests that the SI memory trace was used directly in the comparison task when the retention interval was very short. Second, in experiment 2 , disruption of the SI trace at 300 or $600 \mathrm{msec}$ affected the subsequent comparison made as long as $1500 \mathrm{msec}$ later, indicating that the immediate memory trace in SI was essential for the formation of the longerlasting memory.

Some discrepant observations must be reconciled before the role of SI in tactile working memory can be fully understood. The neural circuits underlying working memory for vibrotactile stimuli have been investigated in detail by Romo et al. (1999), Salinas et al. (2000), Romo and Salinas (2001), and Hernández et al. (2002) in a series of electrophysiological recording studies with monkeys. These authors have identified populations of neurons in SII, the prefrontal cortex, and premotor cortical areas the activity of which differentially encodes the frequency of the first vibration and that sustain this differential activity across the retention interval. However, they did not observe sustained activity among neurons in SI (Salinas et al., 2000), leading them to conclude that neurons in SI do not participate in maintaining the vibrotactile working memory trace. This disagreement with our conclusions may be a result of procedural differences. The monkeys studied by Romo and colleagues were given several months of training on the task, whereas our human subjects were given no previous training at all. Intensity of training could influence the neural mechanisms of working memory. For example, during the course of extensive training given to the monkeys, the temporal entrainment of the SI stimulus representations might have improved (Recanzone et al., 1992a,b), allowing a faster or more efficient transfer of information to "later" cortical areas, such as SII. The contribution of SI to working memory might diminish under these conditions. Additional work will be required to determine whether SI plays a major role in storing information about unfamiliar stimuli and a lesser role in well rehearsed stimuli.

In conclusion, although early sensory cortical areas are commonly viewed as contributing only to the on-line processing and representation of sensory events, the present findings add to a growing body of evidence that these areas also constitute important components of the networks subserving perceptual learning and short- and long-term memory more generally (Kosslyn et al., 2001). As such, they are consistent with a model in which the populations of cortical neurons that explicitly encode sensory information also store that information for subsequent use (Fuster, 2001; Harris et al., 2001a).

\section{REFERENCES}

Baddeley A (1996) The fractionation of working memory. Proc Natl Acad Sci USA 93:13468-13472.

Civardi C, Cantello R, Asselman P, Rothwell JC (2001) Transcranial magnetic stimulation can be used to test connections to primary motor areas from frontal and medial cortex in humans. NeuroImage 14:1444-1453.

Cohen LG, Bandinelli S, Sato S, Kufta C, Hallett M (1991) Attenuation in detection of somatosensory stimuli by transcranial magnetic stimulation. Electroencephalogr Clin Neurophysiol 81:366-376.

Darwin CT, Turvey MT, Crowder RG (1972) An auditory analogue of the Sperling partial report procedure: evidence for brief auditory storage. Cognit Psychol 3:255-267.

Disbrow E, Roberts T, Poeppel D, Krubitzer L (2001) Evidence for interhemispheric processing of inputs from the hands in human S2 and PV. J Neurophysiol 85:2236-2244.

Francis ST, Kelly EF, Bowtell R, Dunseath WJ, Folger SE, McGlone F (2000) fMRI of the responses to vibratory stimulation of digit tips. NeuroImage 11:188-202.

Fuster JM (2001) The prefrontal cortex: an update: time is of the essence. Neuron 30:319-333.

Fuster JM, Jervey JP (1981) Inferotemporal neurons distinguish and retain behaviorally relevant features of visual stimuli. Science 212:952-955.

Goldman-Rakic PS (1996) Regional and cellular fractionation of working memory. Proc Natl Acad Sci USA 93:13473-13480.

Hallett M (2000) Transcranial magnetic stimulation and the human brain. Nature 406:147-150.

Harris JA, Petersen RS, Diamond ME (2001a) The cortical distribution of sensory memories. Neuron 30:315-318.

Harris JA, Harris IM, Diamond ME (2001b) The topography of tactile working memory. J Neurosci 21:8262-8269.

Hernández A, Salinas E, Garcia R, Romo R (1997) Discrimination in 
the sense of flutter: new psychophysical measurements in monkeys. J Neurosci 17:6391-6400.

Hernández A, Zainos A, Romo R (2000) Neuronal correlates of sensory discrimination in the somatosensory cortex. Proc Natl Acad Sci USA 97:6191-6196.

Hernández A, Zainos A, Romo R (2002) Temporal evolution of a decision-making process in medial premotor cortex. Neuron 33:959-972.

Jones EG, Powell TP (1969) Connexions of the somatic sensory cortex of the rhesus monkey. II. Contralateral cortical connexions. Brain 92:717-730.

Killackey HP, Gould HJ III, Cusick CG, Pons TP, Kaas JH (1983) The relation of corpus callosum connections to architectonic fields and body surface maps in sensorimotor cortex of new and old world monkeys. J Comp Neurol 219:384-419.

Kosslyn SM, Ganis G, Thompson WL (2001) Neural foundations of imagery. Nat Rev Neurosci 2:635-642.

Lu Z-L, Williamson SJ, Kaufman L (1992) Behavioral lifetime of human auditory sensory memory predicted by physiological measures. Science 258:1668-1670.

Maldjian JA, Gottschalk A, Patel RS, Detre JA, Alsop DC (1999) The sensory somatotopic map of the human hand demonstrated at $4 \mathrm{~T}$. NeuroImage 10:55-62.

Manzoni T (1984) Callosal mechanism for the interhemispheric transfer of hand somatosensory information in the monkey. Behav Brain Res $11: 155-170$.

Miyashita Y, Chang HS (1988) Neuronal correlate of pictorial shortterm memory in the primate temporal cortex. Nature 331:68-70.

Mountcastle VB, Talbot WH, Sakata H, Hyvarinen J (1969) Cortical neuronal mechanisms in flutter-vibration studied in unanesthetized monkeys: neuronal periodicity and frequency discrimination. J Neurophysiol 32:452-484.

Mountcastle VB, Steinmetz MA, Romo R (1990) Frequency discrimination in the sense of flutter: psychophysical measurements correlated with postcentral events in behaving monkeys. J Neurosci 10:3032-3044.

Münchau A, Bloem BR, Irlbacher K, Trimble MR, Rothwell JC (2002) Functional connectivity of human premotor and motor cortex explored with repetitive transcranial magnetic stimulation. $\mathrm{J}$ Neurosci 22:554-561.

Pascual-Leone A, Walsh V, Rothwell J (2000) Transcranial magnetic stimulation in cognitive neuroscience: virtual lesion, chronometry, and functional connectivity. Curr Opin Neurobiol 10:232-237.

Paus T, Jech R, Thompson CJ, Comaeu R, Peters TM, Evbans AC (1997) Transcranial magnetic stimulation during positron emission tomography: a new method for studying connectivity of the human cerebral cortex. J Neurosci 17:3178-3184.

Penfield W, Rasmussen T (1950) The cerebral cortex of man: a clinical study of localization of function. New York: Hafner.

Petrides M (2000a) Dissociable roles of mid-dorsolateral prefrontal and anterior inferotemporal cortex in visual working memory. J Neurosci 20:7496-7503.

Petrides M (2000b) The role of the mid-dorsolateral prefrontal cortex in working memory. Exp Brain Res 133:44-54.

Postle BR, Berger JS, D'Esposito MD (1999) Functional neuroanatomical double dissociation of mnemonic and executive control processes contributing to working memory performance. Proc Natl Acad Sci USA 96:12959-12964.

Recanzone GH, Merzenich MM, Jenkins WM, Grajski KA, Dinse HR (1992a) Topographic reorganization of the hand representation in cortical area $3 \mathrm{~b}$ owl monkeys trained in a frequency-discrimination task. J Neurophysiol 67:1031-1056.

Recanzone GH, Merzenich MM, Schreiner CE (1992b) Changes in the distributed temporal response properties of SI cortical neurons reflect improvements in performance on a temporally based tactile discrimination task. J Neurophysiol 67:1071-1091.

Robinson CJ, Burton H (1980) Somatotopographic organization in the second somatosensory area of $M$. fascicularis. J Comp Neurol 192:43-67.

Röder B, Spence C, Rösler F (2002) Assessing the effect of posture change on tactile inhibition-of-return. Exp Brain Res 143:453-462.

Romo R, Salinas E (2001) Touch and go: decision-making mechanisms in somatosensation. Annu Rev Neurosci 24:107-137.

Romo R, Hernández A, Zainos A, Salinas E (1998) Somatosensory discrimination based on cortical microstimulation. Nature 392:387-390.

Romo R, Brody CD, Hernández A, Lemus L (1999) Neuronal correlates of parametric working memory in the prefrontal cortex. Nature 399:470-473.

Romo R, Hernández A, Zainos A, Brody CD, Lemus L (2000) Sensing without touching: psychophysical performance based on cortical microstimulation. Neuron 26:273-278.

Ruben J, Schwiemann J, Deuchert M, Meyer R, Krause T, Curio G, Villringer K, Kurth R, Villringer A (2001) Somatotopic organization of human secondary somatosensory cortex. Cereb Cortex 11:463-473.

Salinas E, Hernández A, Zainos A, Romo R (2000) Periodicity and firing rate as candidate neural codes for the frequency of vibrotactile stimuli. J Neurosci 20:5503-5515.

Shoham D, Grinvald A (2001) The cortical representation of the hand in macaque and humans S-I: high resolution optical imaging. J Neurosci 21:6820-6835.

Sperling G (1960) The information available in brief visual presentations. Psychol Monogr 74: No. 498.

Strafella AP, Paus T, Barrett J, Dagher A (2001) Repetitive transcranial magnetic stimulation of the human cerebral cortex induces dopamine release in the caudate nucleus. J Neurosci 21:RC157.

Super H, Spekreijse H, Lamme VAF (2001) A neural correlate of working memory in the monkey primary visual cortex. Science 293:120-124.

Ungerleider LG, Courtney AM, Haxby JV (1998) A neural system for human visual working memory. Proc Natl Acad Sci USA 95:883-890.

Wassermann EM (1998) Risk and safety of repetitive transcranial magnetic stimulation: report on suggested guidelines from the International Workshop on the Safety of Repetitive Transcranial Magnetic Stimulation. Electroencephalogr Clin Neurophysiol 108:1-16.

Whitsel BL, Petrucelli LM, Werner G (1969) Symmetry and connectivity in the map of the body surface in somatosensory area II of primates. J Neurophysiol 32:170-183.

Zhou YD, Fuster JM (1996) Mnemonic neuronal activity in somatosensory cortex. Proc Natl Acad Sci USA 93:10533-10537.

Zhou YD, Fuster JM (2000) Visuo-tactile cross-modal associations in cortical somatosensory cells. Proc Natl Acad Sci USA 97:9777-9782. 